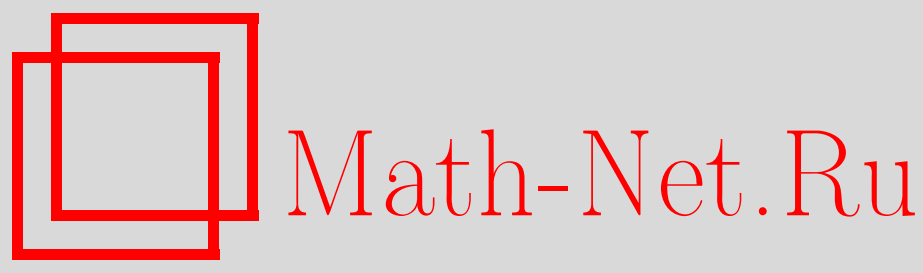

А. Р. Алиев, Э. Х. Эйвазов, О существенной самосопряженности оператора Шредингера в магнитном поле, TMФ, 2011, том 166, номер 2, 266-271

DOI: https://doi.org/10.4213/tmf6608

Использование Общероссийского математического портала Math-Net.Ru подразумевает, что вы прочитали и согласны с пользовательским соглашением http://www.mathnet.ru/rus/agreement

Параметры загрузки:

IP: 44.207 .124 .84

26 апреля 2023 г., 09:48:26

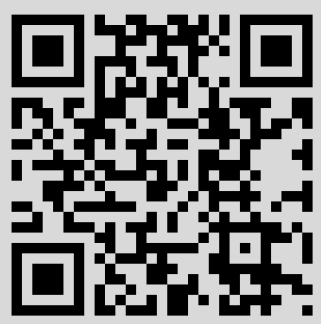




\section{О СУЩЕСТВЕННОЙ САМОСОПРЯЖЕННОСТИ ОПЕРАТОРА ШРЕДИНГЕРА В МАГНИТНОМ ПОЛЕ}

Рассматривается многомерный оператор Шредингера в магнитно-электрическом поле. При определенных условиях типа Штуммеля, налагаемых на магнитный и электрический потенциалы, доказывается существенная самосопряженность магнитного оператора Шредингера.

Ключевые слова: магнитный оператор Шредингера, квантовая механика, существенная самосопряженность, магнитно-электрический потенциал.

\section{1. ВВЕДЕНИЕ}

В настоящей работе в пространстве $L_{2}\left(\mathbb{R}_{n}\right)$ исследуется существенная самосопряженность магнитного оператора Шредингера

$$
H(a, \mathrm{~V})=\sum_{k=1}^{n}\left(-i \frac{\partial}{\partial x_{k}}+a_{k}(x)\right)^{2}+\mathrm{V}(x),
$$

где $\mathrm{V}(x)$ - электрический потенциал, $a(x)=\left(a_{1}(x), a_{2}(x), \ldots, a_{n}(x)\right)$ - магнитный потенциал, причем все потенциалы считаются вещественными. Операторы Шредингера вида (1) играют исключительно важную роль в современной математике, физике и химии, особенно в квантовой механике при исследовании заряженных частиц в магнитном поле и в квантовой химии при изучении магнитооптических свойств молекулярных систем. Как известно (см., например, книгу [1], гл. Х, а также [2]), эволюция квантовой системы, т.е. решение задачи Коши для нестационарного уравнения Шредингера, корректно определена в том и только в том случае, когда гамильтониан этой системы существенно самосопряжен. По этой причине на существенную самосопряженность часто ссылаются как на квантовую полноту. Физический смысл существенной самосопряженности - невозможность для системы уйти на бесконечность за конечное время. Следовательно, установление существенной самосопряженности оператора типа Шредингера должно быть начальной точкой любого дальнейшего исследования соответствующей квантовой системы, в которой

*Бакинский государственный университет, Баку, Азербайджан.

E-mail: alievaraz@yahoo.com, eyvazovelshad@mail.ru

${ }^{\dagger}$ Институт математики и механики НАН Азербайджана, Баку, Азербайджан 
этот оператор используется в качестве гамильтониана. Имеется много работ, посвященных изучению существенной самосопряженности оператора Шредингера в магнитном поле, первая из которых, вероятно, принадлежит Като [3]. Всесторонний обзор результатов о существенной самосопряженности операторов типа (1) можно найти в книгах [1], [4], [5] и в фундаментальной работе [2]. Следует отметить также работы [6], [7], где изучены некоторые спектральные свойства оператора типа (1).

\section{2. ОСНОВНОЙ РЕЗУЛЬТАТ}

Оператор (1) можно записать в виде

$$
H(a, \mathrm{~V})=-\Delta-2 i \sum_{k=1}^{n} a_{k}(x) \frac{\partial}{\partial x_{k}}+q_{1}(x)+q_{2}(x)-i q_{3}(x),
$$

где $\Delta=\sum_{k=1}^{n} \partial^{2} / \partial x_{k}^{2}$ - лапласиан, $q_{1}(x)=\mathrm{V}(x), q_{2}(x)=a^{2}(x)=\sum_{k=1}^{n} a_{k}^{2}(x), q_{3}(x)=$ $\sum_{k=1}^{n} \partial a_{k}(x) / \partial x_{k}$. Предполагается, что измеримые функции $q_{i}(x), i=1,2,3, a_{k}(x)$, $k=1,2, \ldots, n$, удовлетворяют следующим условиям:

a) для всех $i=1,2,3$ функция $q_{i}(x)$ принадлежит пространству Штуммеля $S_{n, \varepsilon}$ (см. работы [1], [5], [8], [9]), т.е. при некотором $\varepsilon \in(0,1)$

$$
\sup _{x \in \mathbb{R}_{n}}\left\{\int_{|x-y| \leqslant 1} \frac{\left|q_{i}(y)\right|^{2}}{|x-y|^{n-4+\varepsilon}} d y\right\}<+\infty
$$

в случае $n \geqslant 4$ и

$$
\sup _{x \in \mathbb{R}_{n}}\left\{\int_{|x-y| \leqslant 1}\left|q_{i}(y)\right|^{2} d y\right\}<+\infty
$$

в случае $n \leqslant 3$;

б) для всех $k=1,2, \ldots, n, a_{k}(x) \in W_{2, \mathrm{loc}}^{1}\left(\mathbb{R}_{n}\right)$ и при некотором $\varepsilon \in(0,1)$

$$
\sup _{x \in \mathbb{R}_{n}}\left\{\int_{|x-y| \leqslant 1} \frac{\left|a_{k}(y)\right|^{2}}{|x-y|^{n-3+\varepsilon}} d y\right\}<+\infty
$$

в случае $n \geqslant 3$ и

в случае $n \leqslant 2$.

$$
\sup _{x \in \mathbb{R}_{n}}\left\{\int_{|x-y| \leqslant 1}\left|a_{k}(y)\right|^{2} d y\right\}<+\infty
$$

Введем в рассмотрение в $L_{2}\left(\mathbb{R}_{n}\right)$ следующие операторы с областью определения $C_{0}^{\infty}\left(\mathbb{R}_{n}\right)$ :

$$
\begin{aligned}
\widetilde{H} \varphi & =\sum_{k=1}^{n}\left(-i \frac{\partial}{\partial x_{k}}+a_{k}(x)\right)^{2} \varphi(x)+\mathrm{V}(x) \varphi(x), \\
\widetilde{H}_{0} \varphi & =-\Delta \varphi ; \quad \widetilde{Q}_{i} \varphi=q_{i}(x) \varphi(x), \quad i=1,2,3 \\
\widetilde{D}_{k} \varphi & =i a_{k}(x) \frac{\partial \varphi}{\partial x_{k}}, \quad k=1,2, \ldots, n, \quad \varphi \in C_{0}^{\infty}\left(\mathbb{R}_{n}\right) .
\end{aligned}
$$

Замыкание оператора $\widetilde{H}_{0}$ обозначим через $H_{0}$.

Нашей целью является доказательство следующего результата.

Теорема. При условиях (а) и (б) оператор $\widetilde{H}$ существенно самосопряжен на $C_{0}^{\infty}\left(\mathbb{R}_{n}\right)$. 


\section{3. ВСПОМОГАТЕЛЬНЫЕ УТВЕРЖДЕНИЯ}

Прежде чем перейти к доказательству теоремы, приведем два вспомогательных утверждения.

ЛЕмма 1. Пусть выполняется условие (а). Тогда симметричные операторы $\widetilde{Q}_{i}$, $i=1,2,3, H_{0}$-ограниченны с $H_{0}$-гранью менъше единиць.

ДоКАЗАТЕЛЬСТво. Покажем, что для достаточно больших положительных $t$ нормы операторов $\widetilde{Q}_{i}\left(H_{0}+t E\right)^{-1}, i=1,2,3$, меньше единицы. Используя идею метода, предложенного в книге [9] (см. стр. 7), имеем

$$
\left\|\widetilde{Q}_{i}\left(H_{0}+t E\right)^{-1}\right\| \leqslant \sqrt{\alpha_{0, i}(t)}, \quad i=1,2,3,
$$

где

$$
\alpha_{0, i}(t)=\sup _{x \in \mathbb{R}_{n}}\left\{\int_{\mathbb{R}_{n}} G(x, y,-t)\left|q_{i}(y)\right|^{2} d y\right\},
$$

$G(x, y,-t)$ - ядро интегрального оператора $\left[\left(H_{0}+t E\right)^{-1}\right]^{2}$.

Случай I: $n=1, n=3$. Используя явный вид ядра $G(x, y,-t)$,

$$
G(x, y,-t)= \begin{cases}\left(\frac{|x-y|}{4 t}+\frac{1}{t^{3 / 2}}\right) e^{-\sqrt{t}|x-y|}, & n=1, \\ \frac{1}{8 \pi t^{1 / 2}} e^{-\sqrt{t}|x-y|}, & n=3,\end{cases}
$$

из выражений (3), (4) и условия (а) заключаем, что существуют пределы

$$
\lim _{t \rightarrow+\infty}\left\|\widetilde{Q}_{i}(H+t E)^{-1}\right\|=0, \quad i=1,2,3 .
$$

Случай II: $n=2$. Из асимптотики функции Ханкеля $H_{0}^{(1)}(z)$ [10]

$$
H_{0}^{(1)}(z) \sim \begin{cases}\frac{2 i}{\pi} \ln z, & z \rightarrow 0, \\ \sqrt{\frac{2}{\pi z}} e^{i(z-\pi / 4)}, & |z| \rightarrow+\infty, \quad-\pi<\arg z<2 \pi,\end{cases}
$$

равенства $G(x, y,-t)=\left((-i / 4) H_{0}^{(1)}(i \sqrt{t}|x-y|)\right)_{-t}^{\prime}$ и условия (а) следует, что выполняются равенства $(5)$.

Случай III: $n \geqslant 4$. В этом случае мы будем использовать оценки Муртазина-Садовничего (см. оценки (5) в книге [9])

$$
\alpha_{0, i}(t) \leqslant\left\{\begin{array}{c}
c \sup _{x \in \mathbb{R}_{n}} \int_{|x-y| \leqslant 1} e^{-\sqrt{2 t}|x-y| / 2}|\ln t| x-y||\left|q_{i}(y)\right|^{2} d y+ \\
+c e^{-\sqrt{2 t} / 4} \sup _{x \in \mathbb{R}_{n}} N_{0, i}(x), \quad n=4, \\
c \sup _{x \in \mathbb{R}_{n}} \int_{|x-y| \leqslant 1} e^{-\sqrt{2 t}|x-y| / 2}|x-y|^{-n+4}\left|q_{i}(y)\right|^{2} d y+ \\
+c e^{-\sqrt{2 t} / 4} \sup _{x \in \mathbb{R}_{n}} N_{0, i}(x), \quad n \geqslant 5,
\end{array}\right.
$$


где

$$
N_{0, i}(x)=\left\{\begin{array}{ll}
\int_{|x-y| \leqslant 1}\left|q_{i}(y)\right|^{2}|\ln | x-y|| d y, & n=4, \\
\int_{|x-y| \leqslant 1}\left|q_{i}(y)\right|^{2}|x-y|^{-n+4}, & n \geqslant 5,
\end{array} \quad i=1,2,3 .\right.
$$

Здесь и в дальнейшем $c$ - любая подходящая, не обязательно все время одна и та же, абсолютная постоянная.

Отметим, что если $|x-y| \leqslant 1$, то для всех $\varepsilon>0$ справедливо неравенство $\mid x-$ $\left.y\right|^{-n+4-\varepsilon} \geqslant|x-y|^{-n+4}$. Поэтому из условия леммы вытекает, что $\sup _{x \in \mathbb{R}_{n}} N_{0, i}(x)<$ $+\infty, i=1,2,3$.

Если $n=4$, то из неравенства (6) в силу известного предела $\lim _{\alpha \rightarrow 0} \alpha^{\varepsilon} \ln \alpha=0$, $\varepsilon>0$, получим

$$
\alpha_{0, i}(t) \leqslant c \frac{1}{t^{\varepsilon}} \sup _{x \in \mathbb{R}_{n}} \int_{|x-y| \leqslant 1} \frac{\left|q_{i}(y)\right|^{2}}{|x-y|^{\varepsilon}} d y+c e^{-\sqrt{2 t} / 4} \sup _{x \in \mathbb{R}_{n}} N_{0, i}(x), \quad i=1,2,3 .
$$

Переходя к пределу при $t \rightarrow+\infty$ в формуле (8) и принимая во внимание условие (a), снова получим выражение (5). Пусть теперь $n \geqslant 5$. Покажем, что для каждого $\eta>0$ существует $\delta>0$ такое, что для всех $t>0$ и $x \in \mathbb{R}_{n}$ справедливы неравенства

$$
\alpha_{0, i}(t) \leqslant \eta+\left(c e^{-\sqrt{2 t} \delta / 2}+c e^{-\sqrt{2 t} / 4}\right) \sup _{x \in \mathbb{R}_{n}} N_{0, i}(x), \quad i=1,2,3 .
$$

Прежде всего заметим, что для любого $\delta>0$ имеет место неравенство

$$
\begin{aligned}
& \int_{|x-y| \leqslant 1} e^{-\sqrt{2 t}|x-y| / 2}|x-y|^{-n+4}\left|q_{i}(y)\right|^{2} d y \leqslant \int_{|x-y| \leqslant \delta}|x-y|^{-n+4}\left|q_{i}(y)\right|^{2} d y+ \\
& \quad+e^{-\sqrt{2 t} \delta / 2} \int_{\delta \leqslant|x-y| \leqslant 1}|x-y|^{-n+4}\left|q_{i}(y)\right|^{2} d y \leqslant \\
& \leqslant \int_{|x-y| \leqslant \delta}|x-y|^{-n+4}\left|q_{i}(y)\right|^{2} d y+e^{-\sqrt{2 t} \delta / 2} \int_{|x-y| \leqslant 1}|x-y|^{-n+4}\left|q_{i}(y)\right|^{2} d y \leqslant \\
& \leqslant \delta^{\varepsilon} \int_{|x-y| \leqslant \delta}|x-y|^{-n+4-\varepsilon}\left|q_{i}(y)\right|^{2} d y+e^{-\sqrt{2 t} \delta / 2} \int_{|x-y| \leqslant 1}|x-y|^{-n+4}\left|q_{i}(y)\right|^{2} d y \leqslant \\
& \leqslant \delta^{\varepsilon} \int_{|x-y| \leqslant 1} \frac{\left|q_{i}(y)\right|^{2}}{|x-y|^{n-4+\varepsilon}} d y+e^{-\sqrt{2 t} \delta / 2} N_{0, i}(x) .
\end{aligned}
$$

Учитывая полученное неравенство и условие (а), получаем оценки (9).

Пусть $\eta>0$ - любое число. Переходя к пределу при $t \rightarrow+\infty$ в неравенствах (9), получим

$$
\lim _{t \rightarrow+\infty} \alpha_{0, i}(t) \leqslant \eta, \quad i=1,2,3 .
$$

Из неравенств (3) и (10) имеем

$$
\lim _{t \rightarrow+\infty}\left\|\widetilde{Q}_{i}\left(H_{0}+t E\right)^{-1}\right\| \leqslant \sqrt{\eta} .
$$

Отсюда и из произвольности положительного числа $\eta$ получаем

$$
\lim _{t \rightarrow+\infty}\left\|\widetilde{Q}_{i}\left(H_{0}+t E\right)^{-1}\right\|=0 .
$$


Так как $H_{0}$-грань операторов $\widetilde{Q}_{i}, i=1,2,3$, равна (см. монографии [1], [5]) $\lim _{t \rightarrow+\infty}\left\|\widetilde{Q}_{i}\left(H_{0}+t E\right)^{-1}\right\|$, получаем, что $H_{0}$-грань этих операторов равна нулю. Лемма 1 доказана.

Лемма 2. Пусть выполняется условие (б). Тогда операторъ $\widetilde{D}_{k}, k=1,2, \ldots, n$, $H_{0}$-ограниченны с $H_{0}$-гранъю менъше единищы.

ДокАЗАТЕЛЬСтво. Покажем, что для достаточно больших положительных $t$ нормы операторов $\widetilde{D}_{k}\left(H_{0}+t E\right)^{-1}, k=1,2, \ldots, n$, меньше единицы. Обозначим через $R_{0, n, k}(-t)$ интегральный оператор с ядром

$$
R_{0, n, k}(x, y,-t)=-2 \pi\left(x_{k}-y_{k}\right) R_{0, n+2}(x, y,-t),
$$

где $R_{0, n}(x, y,-t)$ - ядро интегрального оператора $\left(H_{0}+t E\right)^{-1}$ в $L_{2}\left(\mathbb{R}_{n}\right)$. Используя интегральное представление для ядра $R_{0, n}(x, y,-t)$ [9]

$$
R_{0, n}(x, y,-t)=\frac{1}{(2 \pi)^{n / 2}} \int_{0}^{+\infty} \frac{e^{-t s}}{s^{n / 2}} e^{-|x-y|^{2} /(2 s)} d s
$$

после несложных выкладок получим равенство

$$
R_{0, n, k}(x, y,-t)=\frac{\partial}{\partial x_{k}} R_{0, n}(x, y,-t) .
$$

Используя неравенство

$$
\left\|a_{k}(x) R_{0, n, k}(-t)\right\| \leqslant \sqrt{\left\|a_{k}(x) R_{0, n, k}^{2}(-t) a_{k}(x)\right\|},
$$

можно показать, что для всех $\varepsilon>0$ существует $T_{0}>0$ такое, что при любых $t>T_{0}$ и $k=1,2, \ldots, n$ справедливо неравенство $\left\|a_{k}(x) R_{0, n, k}(-t)\right\|<\varepsilon$. Положим

$$
\beta_{0, k}(t)=\sup _{x \in \mathbb{R}_{n}}\left\{\int_{\mathbb{R}_{n}} G_{n, k}(x, y,-t)\left|a_{k}(y)\right|^{2} d y\right\}, \quad k=1,2, \ldots, n,
$$

где $G_{n, k}(x, y,-t)$ - ядро интегрального оператора $R_{0, n, k}^{2}(-t)$. Так как

$$
G_{n, k}(-t)=R_{0, n, k}^{2}(-t)=\left(R_{0, n, k}(-t)\right)_{-t}^{\prime},
$$

то из выражения (11) имеем

$$
G_{n, k}(x, y,-t)=2 \pi\left(x_{k}-y_{k}\right) R_{0, n+2}^{\prime}(x, y,-t) .
$$

Воспользовавшись интегральным представлением для ядра $R_{0, n+2}(x, y,-t)$, из равенства (12) получим

$$
G_{n, k}(x, y,-t)=\left(x_{k}-y_{k}\right) R_{0, n}(x, y,-t) .
$$

Отсюда, используя явный вид ядра $R_{0, n}(x, y,-t)$, имеем

$$
\left|G_{n, k}(x, y,-t)\right| \leqslant \begin{cases}c \frac{\left|x_{k}-y_{k}\right|}{\sqrt{t}} e^{-\sqrt{t}|x-y|}, & n=1, \\ c\left|x_{k}-y_{k}\right||\ln t| x-y|| e^{-\sqrt{2 t}|x-y| / 2}, & n=2, \\ c \frac{\left|x_{k}-y_{k}\right|}{|x-y|^{n-2}} e^{-\sqrt{2 t}|x-y| / 2}, & n \geqslant 3 .\end{cases}
$$


Из формулы (14) и оценок Муртазина-Садовничего [9] получим

$$
\beta_{0, k}(t) \leqslant \begin{cases}\frac{c}{\sqrt{t}} \int_{-\infty}^{+\infty}\left|a_{k}(y)\right|^{2} d y, & n=1, \\ \frac{c}{\sqrt[4]{t}} \int_{\mathbb{R}_{2}}\left|a_{k}(y)\right|^{2} d y, & n=2, \\ c \sup _{x \in \mathbb{R}_{n}} \int_{|x-y| \leqslant 1} \frac{e^{-\sqrt{2 t}|x-y| / 2}}{|x-y|^{n-3}}\left|a_{k}(y)\right|^{2} d y+ & \\ +c e^{-\sqrt{2 t} / 4} \sup _{x \in \mathbb{R}_{n}} \int_{|x-y| \leqslant 1} \frac{\left|a_{k}(y)\right|^{2}}{|x-y|^{n-3}} d y, & n \geqslant 3 .\end{cases}
$$

Далее, поступая так же, как при доказательстве леммы 1, из формулы (15) заключаем, что

$$
\lim _{t \rightarrow+\infty}\left\|\widetilde{D}_{k}\left(H_{0}+t E\right)^{-1}\right\|=0
$$

Лемма 2 доказана.

\section{4. ДОКАЗАТЕЛЬСТВО ОСНОВНОГО РЕЗУЛЬТАТА}

ДокАЗАТЕЛЬСтво теоРемы. Из лемм 1 и 2 следует, что оператор

$$
Q=\sum_{i=1}^{3} \widetilde{Q}_{i}+\sum_{k=1}^{n} \widetilde{D}_{k}
$$

бесконечно мал по отношению к $H_{0}$, т.е. его $H_{0}$-грань равна нулю. Следовательно, согласно теореме Като-Реллиха [1] оператор

$$
\widetilde{H}=\sum_{k=1}^{n}\left(-i \frac{\partial}{\partial x_{k}}+a_{k}(x)\right)^{2}+\mathrm{V}(x)
$$

самосопряжен в существенном на $C_{0}^{\infty}\left(\mathbb{R}_{n}\right)$.

\section{Список литературы}

[1] М. Рид, Б. Саймон, Методы современной математической физики, т. 2: Гармонический анализ. Самосопряженность, Мир, М., 1978.

[2] М.Ш. Браверман, О. Милатович, М. А. Шубин, УМН, 57:4(346) (2002), 3-58, arXiv: math/0201231.

[3] T. Kato, Israel J. Math., 13:1-2 (1972), 135-148.

[4] Ю.М. Березанский, Разложение по собственным функииям самосопряженных операторов, Наукова думка, Киев, 1965.

[5] Х. Цикон, Р. Фрезе, В. Кирш, Б. Саймон, Операторы Шредингера с приложениями к квантовой механике и глобальной геометрии, Мир, М., 1990.

[6] М. Б. Губайдуллин, Х. Х. Муртазин, ТМФ, 126:3 (2001), 443-454.

[7] М. Б. Губайдуллин, ТМФ, 130:2 (2002), 267-274.

[8] F. Stummel, Math. Ann., 132:2 (1956), 150-176.

[9] Х. Х. Муртазин, В. А. Садовничий, Спектральный анализ многочастичного оператора Шредингера, Изд-во МГУ, М., 1988.

[10] М. Абрамовиц, И. Стиган (ред.), Справочник по специалъным функциям с формулами, графиками и математическими таблицами, Наука, М., 1979. 